\title{
Titanium Silicon Oxide Nanoflower Coated Polyaniline Nanocomposite for Enhancement of Corrosion Protection Performance on Mild Steel
}

\author{
Pebam Sanjeeta Devi, Nimisha Jadon*, Rajeev Jain \\ School of Studies in Environmental Chemistry, Jiwaji University, Gwalior, India
}

Email address:

nimisha09@yahoo.com (N. Jadon)

${ }^{*}$ Corresponding author

\section{To cite this article:}

Pebam Sanjeeta Devi, Nimisha Jadon, Rajeev Jain. Titanium Silicon Oxide Nanoflower Coated Polyaniline Nanocomposite for Enhancement of Corrosion Protection Performance on Mild Steel. Science Journal of Analytical Chemistry. Vol. 6, No. 3, 2018, pp. 25-31.

doi: 10.11648/j.sjac.20180603.12

Received: June 23, 2018; Accepted: July 7, 2018; Published: August 30, 2018

\begin{abstract}
In this study PANI/titanium silicon oxide (PTS) nanoflower coated nanofiber has been synthesized by chemical oxidation method. PANI nanocomposite has been applied as a corrosion protective material which helps in improving the corrosion resistance of various metal oxides such as titanium silicon dioxide which has been renowned as most promising electrode material for corrosion inhibition. The possible interactions between PANI and titanium silicon oxide and morphological characteristics of the synthesized nanocomposite were investigated by FTIR, XRD, TEM and SEM. The anticorrosion performance of different PANI/titanium silicon oxide (PTS) nanocomposites coatings were investigated in $0.5 \mathrm{M}$ $\mathrm{HNO}_{3}$ medium by the potentiodynamic technique and electrochemical impedance spectroscopy (EIS) on mild steel. The most pronounced improvement in anticorrosion property of PANI was obtained by using 15\% PTS composition of synthesized nanocomposite. The performance of this nanocomposite as an anticorrosive material has been investigated through EIS on mild steel.
\end{abstract}

Keywords: Polyaniline, EIS, Nanocomposites, Corrosion Inhibitor, Mild Steel etc

\section{Introduction}

Recently conducting polymer has been considered as promising corrosion inhibitors for metals and anticorrosion coatings [1]. Polyaniline (PANI) is an exceptional conducting polymer by the properties of chemical and environmental stability, its simplistic process and it's gamely controlled doping level, Conductive polymers have received much attention in recent years due to their potential application in sensors [2-7]. Mild steel are broadly used in the field of industry and machinery. To decrease the corroded metal, corrosion inhibition programs are compulsory by the addition of inhibitor to the system for the prevention of corrosion of the metal surface [8]. For the corrosion protection of metal structures against corrosive species epoxy coating has been used extensively. On the other hand epoxy is a solvent based coating and epoxy results in creation of micro pores into coating matrix leading to poor corrosion resistance [9-12]. One of the applications of PANI nanocomposites is corrosion protection. And numerous studies have expose that different PANI nanocomposites can improve the corrosion resistance of different metals (carbon steel, mild steel, carbon steel, aluminium, reinforced steel, cold -rolled steel (CRS), AX91D Mg alloy) [13-22].

Among all metal oxides, titanium silicon dioxide $\left(\mathrm{TiSiO}_{4}\right)$ is recognised as one of the most promising electrode materials for electrochemical corrosion inhibitor. $\mathrm{TiSiO}_{4}$ has a wide band-gap semiconductor, with a high dielectric constant, which upon compression could become an ultrahard material and because of these features make of titanium silicon oxide an interesting nanocomposite for many technological applications. The excellent thermal stability of $\mathrm{TiSiO}_{4}$ nanoparticles can vouch for its application in the investigation and bring about a substantial enhancement in its performance and compact intercalated $\mathrm{Ti}$ layer that effectively interrupted the corrosive ions pathways 


\section{[23-26].}

The aim of this work is to investigate the effect of $\mathrm{PANI} / \mathrm{TiSiO}_{4}$ nanocomposites coatings as anticorrosion performance on mild steel through EIS investigations.

\section{Experimental}

\subsection{Materials}

Commercial titanium silicon oxide nano-powder $<50 \mathrm{~nm}$ (BET), 99.8\% was supplied by Sigma Aldrich product of USA. Aniline, hydrochloric acid ( $\mathrm{HCl})$, ammonium persulfate (APS), ethanol, DMF were of analytical grade and used as received. Supplied distilled water obtained from Milli-Q assembly. Mild steel coupons having percent composition of $\mathrm{C}$ (0.18), Si (0.19), Mn (0.51), P (0.044), S (0.057), Cr (0.14), Ni (0.09), Mo (0.02), $\mathrm{Cu}(0.06), \mathrm{V}$ (less than 0.01) and remaining $\mathrm{Fe}$ were used. The specimens were abraded into the uniform surface successively using the emery papers of $150,180,320$, 400, 600 and 1000 grade till mirror surface. All the mild steel coupons surface were subjected to ultrasonicaton in a solution with acetone and washed with doubled distilled water to degrease before the coating.

\subsection{Synthesis of PANI/TiSiO ${ }_{4}$ Nanocomposites $^{2}$}

The PANI/TiSiO 4 was prepared by sol-gel method through chemical oxidation polymerisation of aniline and $\mathrm{TiSiO}_{4} .1 \mathrm{M}$ of aniline dissolved in $100 \mathrm{~mL}$ of double distilled water in three different conical flasks (A, B and C) and stirred for $1 \mathrm{~h}$ till the aniline was spread uniformly. After that $1 \mathrm{M}$ HCI was added drop wise from the burette to the above mixture and stirred it till appearance of clear solution. Afterwards different concentration of $\mathrm{TiSiO}_{4}$ nanopowder was added to conical flask A ( $5 \%$ of aniline), B (10\% of aniline) and $\mathrm{C}(15 \%$ aniline $)$ with different concentration followed by the addition of $1 \mathrm{M}$ of APS solution drop wise from the burette then keeps the solution for 6 to $12 \mathrm{~h}$ at $10^{\circ} \mathrm{C}$ then filtered washed with distilled water well and at last washed with methanol and collect the residue in a petridish to keep it in the air oven at $70^{\circ} \mathrm{C}$ for $24 \mathrm{~h}$. Similarly for comparison of properties of different percentage PTS1 (5\%), PTS2 (10\%), PTS3 (15\%) of aniline to $\mathrm{TiSiO}_{4}$ was also been prepared.

\subsection{Fabrication of PANI/TiSiO ${ }_{4}$ Composite Coatings on Mild Steel}

The prepared PANI, PTS1, PTS2 and PTS3 nanocomposites powder were crushed. Then $5 \mathrm{mg}$ prepared nanocomposites (PANI, PTS1, PTS2 and PTS3) slurry was prepared with $5 \mathrm{~mL}$ DMF with continuous stirring for $4 \mathrm{~h}$ at room temperature and then in an ultrasonicator for another 20 min at room temperature. After sonication a uniform dispersion of PANI, PTS1, PTS2 and PTS3 nanocomposites in DMF solution is observed respectively. The mild steel coupons were coated with $100 \mu \mathrm{L}$ solution of PANI, PTS1, PTS2 and PTS3 with DMF respectively, and dried for $30 \mathrm{~min}$ at room temperature and kept in an oven at $30^{\circ} \mathrm{C}$ for $3 \mathrm{~h}$ to form a uniform film on mild steel substrates.

\subsection{Characterization of PANI/ $\mathrm{TiSiO}_{4}$ Nanocomposites}

Fourier transformation infrared (FTIR) spectroscopy using $\mathrm{KBr}$ pellets was performed on PERKINELMER to characterize the synthesized nanocomposite. X-ray diffraction (XRD) analysis of the nanocomposite powder was conducted using Rigaku Miniflex600. Then the surface of the previously applied coating was characterized by using SEM (Scanning electron morphology).

Electrochemical Impedance Spectroscopy (EIS), measurements were analysis between the frequency range of $100 \mathrm{~Hz}$ to $10 \mathrm{mHz}$ and an amplitude of $20 \mathrm{mV}$ using an AUTOLAB 112 Potentiostate galvanostate $302 \mathrm{~N}$ (the Netherlands) with software NOVA 1.1. 0.5 $\mathrm{M} \mathrm{HNO}_{3}$ was used as the electrolyte. The coated mild steel coupon area $1 \times 1 \mathrm{~cm}^{2}$ was used as working electrode and $\mathrm{Ag} / \mathrm{AgCl}$ (saturated $\mathrm{KCl}$ ) electrode used as reference electrode. The experimental data was analysed using software NOVA 1.1.1 differently coated mild steel coupon was also recorded their change of OCP with immersion time. The measurements are performed in $0.5 \mathrm{M} \mathrm{HNO}_{3}$ solution at $5^{\circ} \mathrm{C}$.

\section{Results and Discussions}

\subsection{Structure and Characterisation of Nanocomposites Powder FTIR}

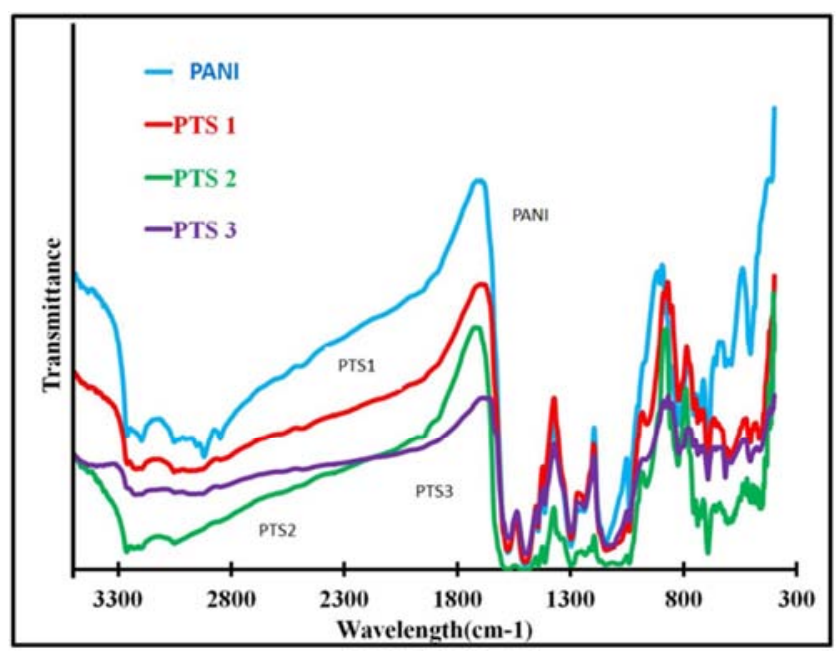

Figure 1. FTIR spectra of PANI, PTS1, PTS2 and PTS3.

Several well defined peaks are observed from the FTIRspectra [Figure 1]. The FTIR spectrum showed peaks at $515.71 \mathrm{~cm}^{-1}$ (C-N-C bonding mode of aromatic), $592.85 \mathrm{~cm}^{-1}$ and $700 \mathrm{~cm}^{-1}$ (C-C, C-H bonding mode of $\pi$ peak aromatic) $831.98 \mathrm{~cm}^{-1}$ (C-H out of plane bonding in benzenoide), $1040.26 \mathrm{~cm}^{-1}$ and $1155.97 \mathrm{~cm}^{-1}$ ( $\mathrm{S}=\mathrm{O}$ bonding) $1505.09 \mathrm{~cm}^{-1}$ (C-N stretching benzoid ring and $1572.52(\mathrm{C}=\mathrm{N}$ - bonding) respectively. The characteristics absorption band of PANI $2900 \mathrm{~cm}^{-1}$ (C-H stretching), $3290 \mathrm{~cm}^{-1}$ (NH stretching for PTS2), $3200 \mathrm{~cm}^{-1}$ (NH stretching for PTS1 and PTS3). Absorption bands of $\mathrm{PANI} / \mathrm{TiSiO}_{4}$ were slightly shifted to 
lower wave numbers when compared with that of PANI. The shift may be due to some electronic interaction between PANI chain and surface of $\mathrm{PANI} / \mathrm{TiSiO}_{4}$.

\section{2. $X R D$}

The XRD patterns of pure PANI, $\mathrm{TiSiO}_{4}$ and different composition of $\mathrm{PANI} / \mathrm{TiSiO}_{4}$ nanocomposites coating are shown in Figure 2. The diffraction peak at $2 \theta=13^{\circ} \mathrm{C}$ and $27^{\circ} \mathrm{C}$ can be attributed to the characteristics peak of PANI, titanium silicon oxide and polyaniline titanium silicon oxide nanocomposites with different composition of titanium silicon oxides, respectively. $2 \theta$ is $28^{\circ}$ for TS at 27 $28^{\circ} \mathrm{C}, 2 \theta$ is $27^{\circ}$ for PTS 1 at $27^{\circ} \mathrm{C}, 2 \theta$ is $29^{\circ}$ for PTS2 at $28^{\circ} \mathrm{C}, 2 \theta$ is $30^{\circ}$ for PTS 3 at $28^{\circ} \mathrm{C}$ and $2 \theta$ of PANI is $29^{\circ}$ at $30^{\circ} \mathrm{C}$. The broad peak of PANI become sharper on increasing the percentage of titanium silicon oxide (PTS1, PTS2 and PTS3) in the composites and shows the amorphous in nature. As the result indicated that $2 \theta$ value of nanocomposite shifted as the concentration of $\mathrm{TiSiO}_{4}$ increases peak become sharper which concluded that the surface of PANI covered by $\mathrm{TiSiO}_{4}$

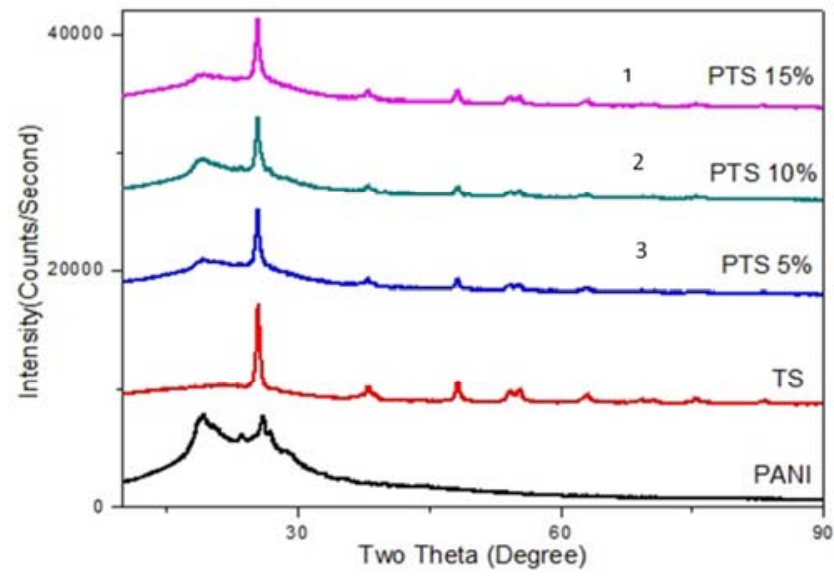

Figure 2. XRD spectra of the prepared PANI materials, different composition polyaniline titanium silicon oxide nanocomposites and titanium silicon oxide (PTS1, PTS2, PTS3) and TS (titanium silicon oxide).

\subsection{Surface Morphological Studies}

All the mentioned composites $\mathrm{TiSiO}_{4}$, PANI and $\mathrm{PANI} / \mathrm{TiSiO}_{4}$ were synthesized in situ with single step chemical oxidative polymerization. Figure 3 shows the images of the electrodes, recorded for mild steel. Figure 3(1) represents SEM for mild steel which shows clear visible polishing marks on the surface. Figure 3(B), represents SEM for $\mathrm{TiSiO}_{4}$, Figure 3(2), represents SEM for PANI and Figure 3(3), and represents SEM for $\mathrm{PANI} / \mathrm{TiSiO}_{4}$. Figure 3(4) represent SEM for mild steel after running EIS in $0.5 \mathrm{M}$ $\mathrm{HNO}_{3}$. This SEM images shows that the morphology of the polyaniline polymer is similar to the nodular structure formed by the aggregation of spherical grains.
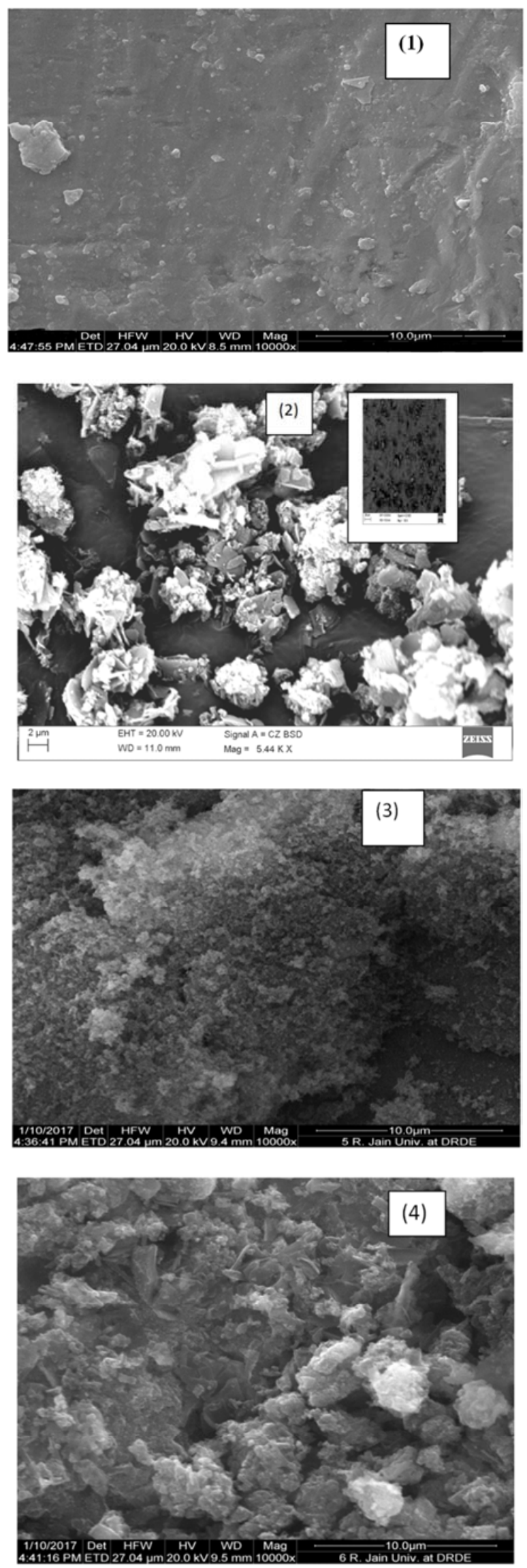

Figure 3. SEM micrographs of (1) bare steel (2) Polyaniline (3) titanium silicon oxide (4) PANI/ titanium silicon oxide. 


\subsection{Transmission Electron Microscopy (TEM)}
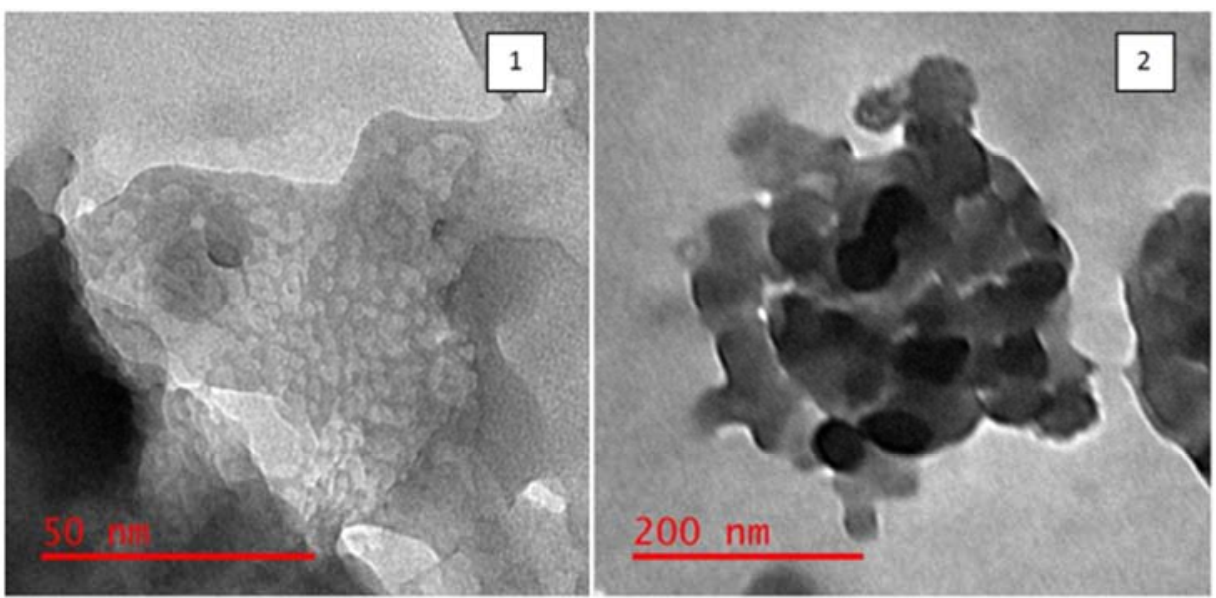

Figure 4. TEM images of (1) Polyaniline (2) Polyaniline titanium silicon oxide.

Figure 4, TEM images shows the (A) Polyaniline (B) Polyaniline titanium silicon oxide. TEM studies were performed on a $120 \mathrm{kv}$ JEOL (model JEM-1230 Electron Microscope). The TEM image of polyaniline shows the formation of PANI nanoparticles in $50 \mathrm{~nm}$ which is attributed to use of ultrasonic irradiation during synthesis Figure 4(1). TEM images of polyaniline titanium silicon oxide nanocomposites confirm the uniform distribution of titanium silicon oxide in PANI Figure 4(2). Also it has been established titanium silicon oxide are finely dispersed in PANI matrix measured in $200 \mathrm{~nm}$. Furthermore it has been clearly observed that the improvement of uniformly dispersion of increased concentration of titanium silicon oxide during polymerisation into PANI matrix.

\subsection{Corrosion Resistance of Coatings}

EIS measurements

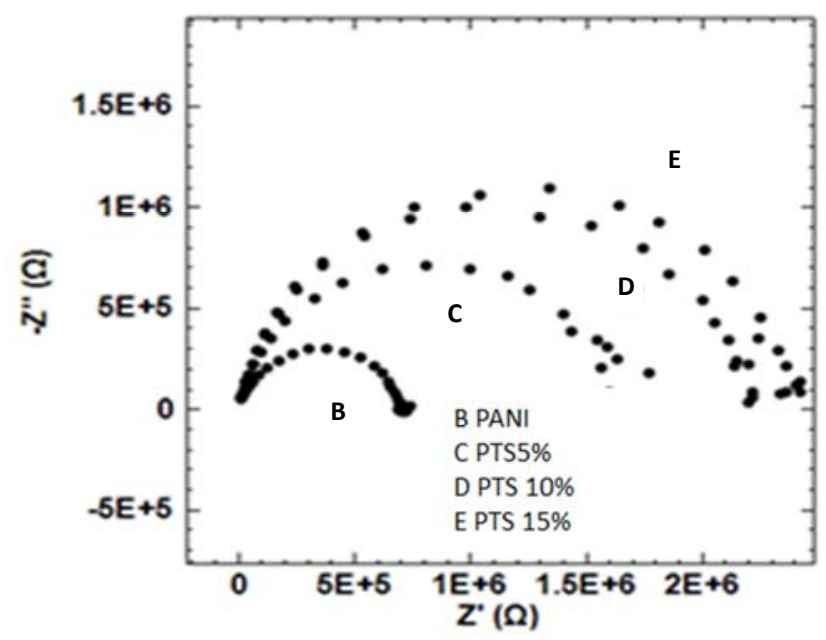

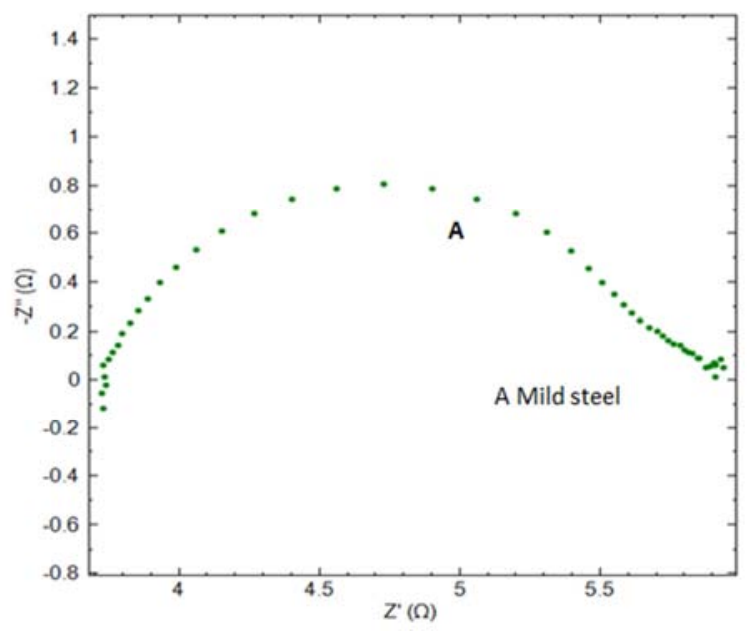

Figure $5(1)$.

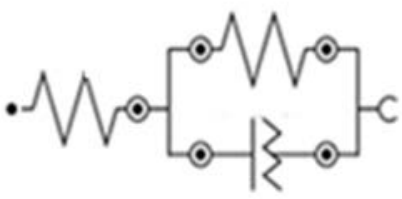

Fig: a

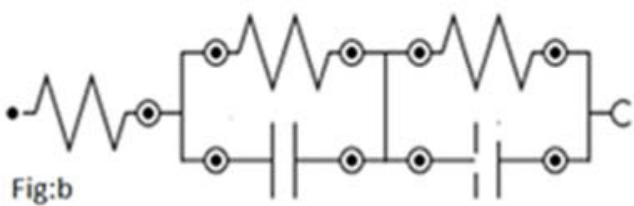

Figure 5 (2)

Figure 5. (1). EIS plots of (a) mild steel and coated mild steel with PANI and different concentration of (b) PANI, (c) PTS1, (d) PTS2 and (e) PTS3 immersion for $1 \mathrm{~h}$ at $5^{\circ} \mathrm{C}$. Figure 5 (2) is electrical equivalent circuits used for modelling the impedance results of EIS of PANI and PTS3. 
Table 1. Electrochemical Impedance Parameters at $5^{\circ} \mathrm{C}$.

\begin{tabular}{lllll}
\hline $\begin{array}{l}\text { Conc. (1mg } \\
\text { nanocomposites per } \\
\text { 1mL of DMF) }\end{array}$ & $\mathbf{R}_{\mathrm{ct}}\left(\mathbf{\Omega} \mathbf{~ c m}^{-2}\right)$ & $\mathbf{f}_{\text {max }}(\mathbf{H z})$ & $\mathbf{C}_{\mathrm{dl}}\left(\mathbf{F c m}^{-2}\right)$ & $\mathbf{I . E . ~ ( \% )}$ \\
\hline Mild Steel & 5.29 & 115.14 & 0.00026 & - \\
PANI & 22.54 & 0.54 & 0.013 & 76.53 \\
PTS1 & $12.32 \times 10^{3}$ & 15.99 & $2.39 \times 10^{-6}$ & 99.95 \\
PTS2 & $119.08 \times 10^{7}$ & 49.417 & $2.7059 \mathrm{E}-12$ & 99.99 \\
PTS3 & $336.16 \times 10^{7}$ & 65.513 & $7.23047 \mathrm{E}-13$ & 99.99 \\
\hline
\end{tabular}

Electrochemical impedance measurements were used for analysis of corrosion inhibition of polyaniline nanocomposites coatings. EIS technique was applied to investigate the electrode/electrolyte interface and corrosion processes that occur on mild steel surface in the presence and absence of PANI and PTS nanocomposites. To ensure complete characterization of the interface and surface processes, EIS measurements were made at OCP in a wide frequency range at $5^{\circ} \mathrm{C}$. Figure 5 (1) shows Nyquist plots for mild steel electrode and coated mild steel by $\mathrm{PANI} / \mathrm{TiSiO}_{4}$ immersed in $0.5 \mathrm{M} \mathrm{HNO}_{3}$ solution at $5^{\circ} \mathrm{C}$ in absence and presence of various concentrations of the nanocomposites. It is cleared from the Figure 5(1) that the diameter of the semicircle increases with the increase in concentration of the extract, indicating an enhancement of corrosion resistance capacity of metal. Also Figure 5(2) shows the fitting of mild steel and PTS3, the fitting of uncoated of mild steel and the electrochemical circuit fitting of coated mild steel by PTS3.

In case of impedance measurement, the inhibition efficiency was evaluated from the measured charge transfer resistance (Ret) value as

$$
I \%=\frac{R_{e t}^{\prime}-R_{e t}}{R_{e t}^{\prime}} \times 100
$$

Where $R_{\text {et }}$ and $R_{\text {et }}$ are the charge transfer resistance value in the absence and presence of inhibitors.

The value of electrochemical double layer capacitance $\left(\mathrm{C}_{\mathrm{dl}}\right)$ was calculated at the frequency, $\mathrm{f}_{\max }$ using the following equation

$$
\mathrm{C}_{\mathrm{dl}}=1 / 2 \pi \mathrm{f}_{\max } \mathrm{R}_{\mathrm{ct}}
$$

Where, $f_{\max }$ is the frequency at which the imaginary component of the impedance is maximal.

The impedance data listed in the Table 1 indicate that the values of both $R_{c t}$ and I.E \% are found to increase with increase in concentration of the $\mathrm{PANI} / \mathrm{TiSiO}_{4}$, while the values of $\mathrm{C}_{\mathrm{dl}}$ are found to decrease.

\subsection{Potentiodynamic Polarization}

Potentiodynamic polarization curves for mild steel in 0.5 $\mathrm{M} \mathrm{HNO}_{3}$ solutions in absence and presence of various concentrations of PANI and PTS nanocomposites at $5^{\circ} \mathrm{C}$ are shown in Figure 3 (a). The extrapolation of Tafel straight line allows the calculation of the corrosion current density ( $\left.i_{\text {corr }}\right)$. The values of $i_{\text {corr }}$, the corrosion potential $\left(E_{\text {corr }}\right)$, cathodic and anodic Tafel slopes $\left(\beta_{\mathrm{c}}\right.$ and $\left.\beta_{\mathrm{a}}\right)$ and the percentage of inhibition efficiency (I.E.\%) are given in the Table 2.

Inspections of the data in Figure 6 reveal that at a given temperature, the addition of the PANI and PTS nanocomposites to the acid solution increases both the anodic and cathodic overpotentials, decreases the corrosion current density ( $\left.\mathrm{i}_{\text {corr }}\right)$. The change in cathodic and anodic Tafel slopes $\left(\beta_{\mathrm{c}}\right.$ and $\beta_{\mathrm{a}}$ ) shown in the Table 2 indicates that adsorption of PANI and PTS nanocomposites modify the mechanism of the anodic dissolution as well as cathodic hydrogen evolution. From Figure 6, it is cleared that both cathodic and anodic reactions are inhibited and the inhibition increases as the inhibitor concentration increases in acid media, but the anode is more polarized, indicating the process of metal dissolution is more inhibited. From Table 2, it is also cleared that there is no definite trend in the shift of $\mathrm{E}_{\text {corr }}$ values, in presence of various concentration of the extract in $0.5 \mathrm{M} \mathrm{HNO}_{3}$ solutions. This result indicates that PANI and PTS nanocomposites can be classified as mixed type of inhibitor in $0.5 \mathrm{M} \mathrm{HNO}_{3}$ solutions. Figure 6 shows the tafel plot of (a) uncoated mild steel, coated mild steel by (b) PANI (c) PTS1 (d) PTS2 and (e) PTS3 nanocomposites.

Inhibition Efficiency (IE \%) was calculated using the relationship

$$
I E \%=\frac{I_{\text {corr }}-I_{\text {corr }(\text { inh })}}{I_{\text {corr }}} \times 100
$$

Where $I_{\text {corr }}$ and $I_{\text {corr (inh) }}$ represent the corrosion current

\begin{tabular}{|c|c|c|c|c|c|c|}
\hline Temp (K) & Conc. (1mg nanocomposites per $1 \mathrm{~mL}$ of DMF) & $-E_{\text {corr }}(\mathrm{mV}$ vs. SCE) & $b_{a}(m V /$ decade $)$ & $b_{c}(m V /$ decade $)$ & $I_{\text {corr }}\left(\mathrm{mA} \mathrm{cm} \mathrm{cm}^{-2}\right)$ & I.E. (\%) \\
\hline \multirow{5}{*}{$5^{\circ} \mathrm{C}$} & Mild Steel & 420.7 & 510.95 & 292.54 & 14.619 & - \\
\hline & PANI & 422.08 & 210.82 & 172.38 & 3.70 & 74.67 \\
\hline & PTS 1 & 427.87 & 149.65 & 231.82 & $95 \times 10^{-6}$ & 99.99 \\
\hline & PTS2 & 430.62 & 133.30 & 195.90 & $52.48 \times 10^{-6}$ & 99.99 \\
\hline & PTS3 & 439.38 & 54.75 & 97.12 & $126 \times 10^{-6}$ & 99.99 \\
\hline
\end{tabular}
density values without and with inhibitor, respectively.

Table 2. Potentiodynamic polarization parameters for the corrosion of mild steel. 


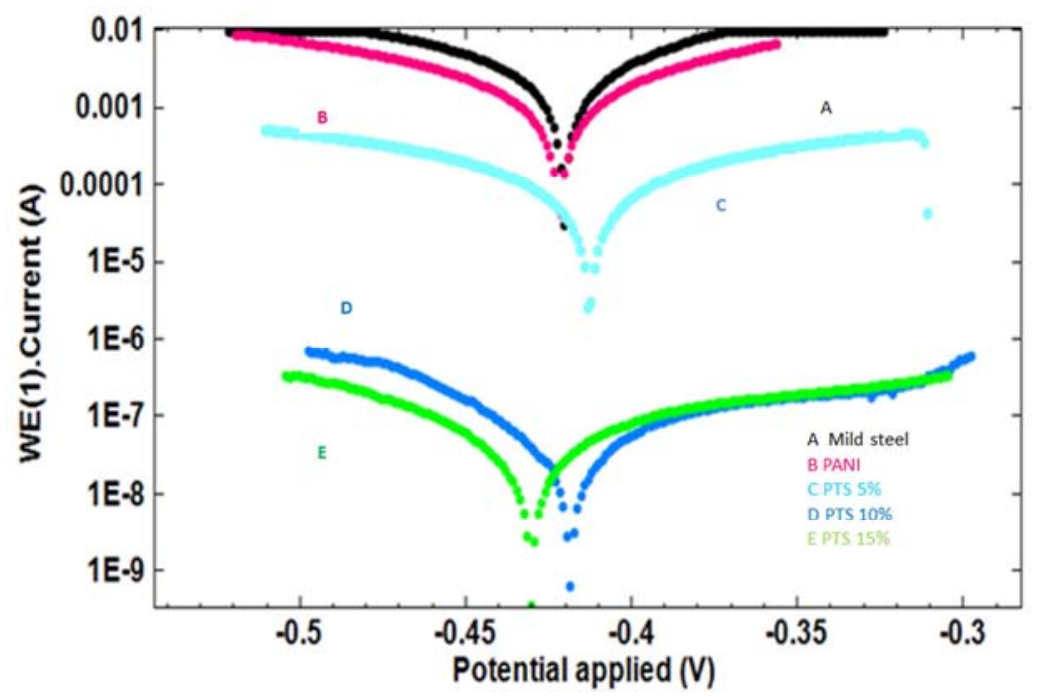

Figure 6. Tafel plots for a (a) uncoated mild steel, coated mild steel by (b) PANI, (c) PTS1, (d) PTS2, (e) PTS3 nanocomposites samples.

\section{Conclusion}

PANI, PTS1, PTS2 and PTS3 nanocomposites are synthesized in the presence of PANI and titanium silicon oxide by using oxidative polymerization of aniline with ammonium sulphate (APS). The synthesized nanocomposites are characterized by SEM, FTIR and XRD. SEM morphology shows a unique hierarchical morphology of the polyaniline polymer is similar to the nodular structure formed by the aggregation of spherical grains of $\mathrm{TiSiO}_{4}$. By using potentiodynamic polarization measurements the coated mild steel with PANI, PTS1, PTS2 and PTS3 is exposed to $0.5 \mathrm{M}$ $\mathrm{HNO}_{3}$ for the corrosion protection performance has been investigated. Corrosion inhibition efficiency has been calculated from EIS and tafel data. It is confirmed from the parametric estimation that the PANI, PTS1, PTS2 and PTS3 nanocomposites with DMF coating formulation shows the higher anticorrosion performance. PANI, PTS1, PTS2 and PTS3 nanocomposites showed good corrosion inhibition efficiency with mixed type inhibitor.

\section{Acknowledgements}

This work was supported by Jiwaji University, Gwalior (M.P.) India [310, 2016]. We acknowledge Prof. P. Rajaram for the TEM facility, SOS in Physics, Jiwaji University Gwalior, and M.P. India 474011 and we also would like to acknowledge for the SEM facility to the SEM laboratory, Geological Survey of India Kolkatta.

\section{References}

[1] E. E. Oguzie, Corros. Sci 50 (2008) 2993-2998.

[2] H. A. Sorkhabi, E. Asghari, Electrochem. Acta. 54 (2008) 1578-1583.

[3] A. Ostav ari, S. M. Hoseinieh, M. Peikari, S. R. Shadizadeh, S. J. Hashemi. Corros. Sci. 51 (2009) 1935-1949.
[4] Y. Zhao, Z. Zhang, L. Yu, React. Funct. Polym. 102 (2016) 20-26.

[5] S. S. Umare, B. H. Shambhakar, J. Appl. Polym. Sci. 127 (2013) 3349-3355.

[6] S. Dhibar, P. Bhattacharya, G. Hatui, S. Sahoo, C. K. Das, ACS Sustain. Chem. Eng. 2 (2014) 1114-1127.

[7] R. Jain, N. Jadon, A. Pawaiya, TrAC 97(2017) 363-373.

[8] J. Prokes, J. Stejskal, Polym. Degrad. Stab. 86 (2004) 187-195.

[9] E. Armelin, C. Aleman, J. J Iribarren, Prog. Org. Coat. 65 (2009) 88-93.

[10] A. B. Samui, A. S. Patankar, J. Rangaranjan, P. C. Deb, Prog. Org. Coat. 47 (2003) 1-7.

[11] R. Karthikaiselvi, S. Subhashini, JAAUBAS, 16 (2014) 74-82.

[12] I. Zaman, T. T. Phan, H. C. Kuan, Q. S. Meng, L. T. B. La, L. Luong, O. Youssf, J. Ma, Polym. 52 (2011) 1603-1611.

[13] E. Matin, M. M. Attar, B. Ramezanzadeh, Prog Org Coat 78 (2015) 395-403

[14] Y.-J. Wan, L.-C. Tang, L.-X. Gong, D. Yan, Y.-B. Li, L.-B. Wu, J.-X. Jiang, G.-Q. Lai, Carbon 69 (2014) 467-480.

[15] H. Yi, C. L. Chen, F. Zhong, Z. H. Xu, Polym. 26 (2014) 255264.

[16] V. A. Mooss, A. A. Bhopale, P P. Deshpande, A. A. Athawale, Chemical Papers, (2017) 1-14.

[17] S. Niladri, S. Gyanaranjan, D. Rashmita, P. Gyanaranjan, S. Deepak, S. K. Sarat, Ind. Eng. Chem. Res. 55 ( 2016) 29212931.

[18] S. S Umare, B. H. Shambharkar, J. Appl. Polym. Sci. 127 (2013) 3349-3355.

[19] B. A. Abd-El-Nabey, O. A. Abdullatef, G. A. El-Naggar, E. A. Matter, R. M. Salman, Int. J. Electrochem. Sci. 11(2016) 2721-2733.

[20] Z. Yunyan, Z. Zhiming, Y. Liangmin, React. Funct. Polym. 102 (2016) 20-25. 
[21] Z. Yingjun, S. Yawei, Z. Tao, M. Guozhe, W. Fuhui, Prog. Org. Coat. 76 (2013) 804- 811.

[22] J. J. Fang, K. Xu, L. H. Zhu, Zh. X. Zhou, H. Q. Tang, Corros. Sci. 49 (2007) 4232-4242.

[23] Yilser Devrim, Serdar Erkan, Nurcan Bac, Inci Eroglu, Int. J. Hydrogen Energy 37 (2012) 16748-16758
[24] A. U. Chaudhry, B. Mansoor, T. Mungole, G. Ayoub, D. P. Field, Electrochim. Act. 264 (2018) 69-82.

[25] N. N. Taheri, B. Ramezanzadeh, M. Mahdavian, G. Bahlakeh, J. Ind. Eng. Chem. 63 (2018) 322-339.

[26] B. Ramezanzadeh, G. Bahlakeh, M. Ramezanzadeh, Corros. Sci. 137 (2018) 111-126. 